2

3

4

5

6

7

8

9

10

11

12

13

14

15

16

17

18

19

20

21

22

23

24

25

26

27

28

29 30 0

8

\title{
Comparative analysis of genome-encoded viral sequences reveals the evolutionary history of Flaviviridae.
}

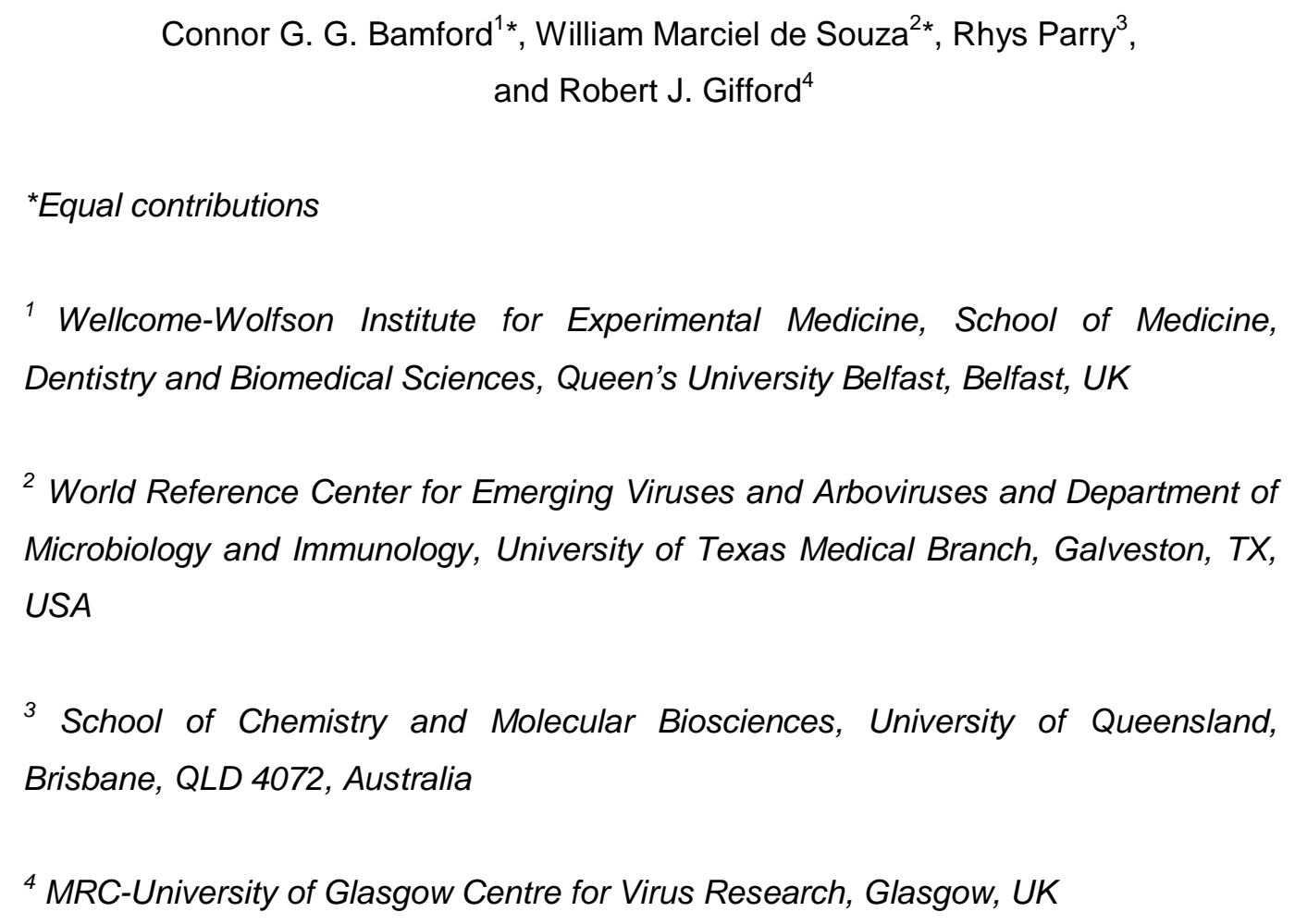

${ }^{2}$ World Reference Center for Emerging Viruses and Arboviruses and Department of Microbiology and Immunology, University of Texas Medical Branch, Galveston, TX, USA

3 School of Chemistry and Molecular Biosciences, University of Queensland, Brisbane, QLD 4072, Australia

${ }^{4}$ MRC-University of Glasgow Centre for Virus Research, Glasgow, UK

\section{Corresponding author:}

3 Robert J. Gifford,

4 MRC-University of Glasgow Centre for Virus Research, 464 Bearsden Rd, Bearsden, Glasgow G61 1QH

6 E-mail: robert.gifford@glasgow.ac.uk

9 Keywords: Evolution | Virus | Flavivirus | Paleovirology | Genomics | 


\section{Abstract}

2

The flaviviruses (family Flaviviridae) are a group of positive-strand RNA

3 viruses, many of which pose serious risks to human health on a global scale. Here,

4 we calibrate the timeline of flavivirus evolution using flavivirus-derived DNA

5 sequences identified in animal genomes. We demonstrate that the family is at least

6100 million years old and show that this timing can be integrated with dates inferred

7 from co-phylogenetic analysis and paleontological records to produce a cohesive

8 overview of flavivirus evolution in which the main subgroups originate early in animal

9 evolution and broadly co-diverge with animal phyla. In addition, we show that the

10 arthropod-borne 'classical' flaviviruses first evolved from tick-specific viruses, and

11 only later adapted to become insect-borne. Our findings demonstrate that the

12 biological properties of flaviviruses have been acquired over many millions of years

13 of evolution, implying that broad-scale comparative analysis can reveal fundamental

14 insights into flavivirus biology. We implement a novel approach to computational

15 genomic studies of viruses that can support these efforts by enabling more efficient

16 utilization of evolution-related domain knowledge in virus research.

17

18

22

23

\section{Significance}

Understanding how pathogenic viruses evolved can provide vital insights into their biology. In this study we use genomic data to show that flaviviruses - a group of viruses that includes important pathogens such as Dengue virus and hepatitis $C$ virus - arose through an extended history of evolutionary interaction with host and vector species. Our findings show that comparative studies can productively utilise genomic data to reveal insights into flavivirus biology, which will help to facilitate the development of more effective antiviral treatments and strategies. 
1

2

3

4

5

\section{Introduction}

The flaviviruses (family Flaviviridae) are a diverse group of enveloped, positive-strand RNA viruses, many of which cause disease in humans and domestic animals. Arthropod-borne flaviviruses such as Zika virus (ZIKV), Dengue virus (DENV), and yellow fever virus (YFV) are the causative agents of large-scale outbreaks that result in millions of human infections every year, while the bloodborne hepatitis $\mathrm{C}$ virus (HCV) is a major cause of chronic liver disease $[1,2]$.

Currently, four flavivirus genera are recognised: Pegivirus, Pestivirus, Hepacivirus and Flavivirus [3]. These genera contain viruses that have monopartite genomes $\sim 10$ kilobases $(\mathrm{Kb})$ in length and encoding one or more large polyproteins that are co- and post-translationally cleaved to generate mature virus proteins. The structural proteins of the virion - capsid $(\mathrm{C})$, premembrane (prM) and envelope (E) are encoded toward the 5' end of the genome, while genes encoding non-structural (NS) proteins are located further downstream [4].

A diverse variety of novel 'flavivirus-like' virus species have been described over recent years, reflecting progress in the use of metagenomic approaches to broadly survey the virome. Most of these newly identified viruses have yet to be incorporated into official taxonomy [5-9]. They exhibit a much greater range of variation in genome structure than is found among representatives of officially recognised flavivirus genera, with genome sizes ranging up to $20 \mathrm{~Kb}$ [10], and one novel group - the Jingmenviruses (JMVs) - comprises viruses with genomes that are segmented rather than monopartite [11].

To prevent the spread of pathogenic viruses, it is helpful to understand their evolutionary history in as much detail as possible, because this can provide crucial insights into virus biology and host-virus relationships including mechanisms of pathogenesis and likelihood of zoonosis. However, knowledge of the appropriate evolutionary timescale is critical. So far, most studies of flavivirus evolution have proposed relatively short timelines in which individual flavivirus genera and sub- 
1 groups emerge within the last 10-100 thousand years [12]. However, these studies

2 were based on viruses sampled from a relatively restricted range of animal hosts. By

3 contrast, more recent studies utilising metagenomic techniques to sample flavivirus

4 diversity across a broad range of animal species have prompted suggestions of a

5 much longer timeline extending over hundreds of millions of years [6]. Unfortunately,

6 due to time-dependent effects on viral evolutionary rates [13, 14], it is not possible to

7 discriminate between these starkly contrasting possibilities using genome data

8 sampled from contemporary flaviviruses.

'Endogenous viral elements' (EVEs) are virus-derived DNA sequences found in the genomes of metazoan species [15]. EVEs derived from RNA viruses are

11 thought to originate when infection of germline cells leads to the generation of viral 12 cDNA and its subsequent integration into chromosomal DNA (presumably mediated 13 by retroelement activity) [16]. The integrated viral genes can be inherited as host 14 alleles and sometimes persist in the gene pool long enough to become genetically 15 fixed (i.e. reach a frequency of $100 \%$ in the species gene pool). These fixed EVEs 16 often derive from viruses that circulated millions of years ago and thereby provide a 17 rare glimpse into the distant history of virus evolution. EVEs derived from flaviviruses, 18 which we refer to here as 'endogenous flaviviral elements' (EFVs), have been 19 identified in some arthropod species including insects $[15,17,18]$ and crustaceans [7]. Interestingly, some of these - most notably those found in the genomes of Aedes

21 mosquitoes - have been shown to produce PIWI-interacting RNAs (piRNAs) in the antisense orientation, suggesting they might provide the basis for a form of heritable antiviral immunity analogous to that of CRISPR-Cas [16, 18-20]. Here, we use comparative approaches to investigate the long-term evolutionary history of flaviviruses, making extensive use of the EVE 'fossil record'. We show that although EFV sequences have only rarely been fixed in the animal germline, those that have 27 represent a broad diversity of flavivirus groups. Moreover, incorporating these 28 sequences into a comprehensive phylogenetic analysis of the Flaviviridae enables 
1 calibration of the timeline of flavivirus evolution and revises current understanding of

2 flavivirus evolution. To facilitate the downstream use of the evolution-related domain

3 knowledge generated in our study, we published our data and analyses in the form of

4 a cross-platform, interactive database that can be used to reproduce our analyses

5 and facilitates re-use of the associated data items.

6

\section{RESULTS}

8 Creation of open resources for reproducible genomic analysis of flaviviruses

Comparative genomic analyses generally entail the construction of complex data sets comprising molecular sequence data linked to other kinds of information.

11 Usually these include genome feature annotations and multiple sequence alignments 12 (MSAs) as well as other diverse kinds of data. We used GLUE - an open, platform13 independent software toolkit for storage and interpretation of sequence data [21] - to 14 develop an openly accessible online resource for comparative genomic analysis of flaviviruses (Flavivirus-GLUE) that preserves the 'state' of our data so that our analyses can be precisely and widely replicated (Fig. S1a-b). Furthermore, hosting of

17 the Flavivirus-GLUE project in an openly accessible online version control system (GitHub) provides a platform for ongoing development of this resource by multiple collaborators, following practices established in the software industry (Fig. S1c). in comparative sequence analysis of flaviviruses, including: (i) a set of reference sequences representing all known flavivirus species (Table S1); (ii) sequence and isolate-specific information (e.g. host species, vector species) in tabular form; (iii) a standardized set of flavivirus genome features and their coordinates within selected reference genome sequences; (iv) a set of MSAs incorporating all sequences in the project. Loading the project into the GLUE 'engine' generates a relational database 27 that not only contains the data items associated with our analysis, but also 28 represents the complex semantic links between them (Fig. S1a). The Flavivirus- 
1 GLUE project can be installed on all commonly-used computing platforms and is also

2 fully containerised via Docker [22]. Reproducible comparative analysis can be

3 implemented within the Flavivirus-GLUE project using GLUE's command layer, which

4 can coordinate interactions between the relational database and bioinformatics

5 software tools (Fig. S1b). These interactions can leverage 'structured query

6 language' (SQL) - the standard user and application program interface of relational

7 databases - to provide a high degree of flexibility and power over the way in which

8 data are retrieved.

9

10 All major flavivirus lineages are represented in the genomic 'fossil record'

11 We incorporated EFVs into our Flavivirus-GLUE project so that the unique

12 information contained in these sequences could be utilised in comparative analysis.

13 To identify EFVs, we performed systematic in silico screening of whole genome

14 sequence (WGS) data representing 1075 animal species and identified 374 EFV loci

15 in 36 species (Table 1, Table S1). We constructed multiple sequence alignments

16 (MSAs) of groups of EFV sequences putatively derived from a single germline

17 incorporation event (i.e. orthologs and duplicated elements) and generated

18 representative EFV consensus sequences from these (Fig. 1, Fig. S2). We used

19 MSAs containing EFV sequences as input for maximum likelihood phylogenetic

20 analysis to reconstruct the evolutionary relationships between EFVs and

21 contemporary flaviviruses (Fig. 2, Fig S3). EFVs were assigned unique identifiers

22 that (i) capture information about the taxonomic group of viruses they are derived

23 from, (ii) uniquely identify the germline incorporation event and genomic locus

24 associated with each sequence, and (iii) indicate the distribution of related

25 sequences across host species (Fig. 1). EFV locus and consensus sequences,

26 MSAs, and all associated metadata were incorporated into Flavivirus-GLUE. We

27 extended the database schema of Flavivirus-GLUE to capture the semantic 
1 relationships between these data items, so that they can be interactively accessed

2 via the underlying database.

Phylogenetic reconstructions show that the Flaviviridae are clearly split into

4 two major lineages - "pegi-hepaci" and "flavi-pesti" - each of which contains several

5 well-supported subgroups [9]. EFVs derived from each of these major flavivirus

6 lineages and subgroups are represented in the genomic 'fossil record'. A single EFV

7 that derives from the "pegi-hepaci" lineage was identified in the genome of a marine

8 mollusc - the Eastern emerald elysia (Elysia chlorotica), demonstrating that the host

9 range of these viruses extends to invertebrates (Fig 2a). However, all other EFVs

10 identified in our screen grouped with the "flavi-pesti" lineage, and these included

11 representatives of diverse "flavi-like" and "pesti-like" groups (Fig $\mathbf{2 b - g}$, Fig S3). In

12 addition, an EFV recovered from the genome of a priapulid worm (Priapulus

13 caudatus) appears to represent a novel subgroup, here labelled ' $\mathrm{X} 1$ ', within the "flavi-

14 like" lineage (Fig 2b).

16 Fixation of flavivirus-derived elements in the animal germline is rare

17 Of the 1075 species screened in our analysis only 36 were found to harbour

18 EFVs in their genomes, but those that did often contained multiple distinct EFV loci.

19 We estimate these EFVs to represent at least 25 distinct germline colonisation

20 events. The low number of events relative to sequences reflects our decision to

21 conservatively assume that the presence of multiple EFV loci in individual species or

22 taxonomic groups reflects intragenomic amplification rather than multiple

23 independent germline incorporation events. The number of events could potentially

24 be much higher if distinct germline incorporation events by closely related viruses

25 have occurred during the evolution of these species. Notably, however, where

26 multiple EFV sequences occur, they generally comprise contiguous and/or

27 overlapping fragments of larger genome segments and in many cases, the

28 overlapping segments are nearly identical at nucleotide level (Fig. S2). This suggests 
1 that longer cDNA sequences may been fixed in the germline initially before

2 subsequently being duplicated and/or fragmented.

The largest set of EFV sequences putatively derived from a single germline

4 incorporation event is found in the insect superfamily Apoidae (bees and sphecoid

5 wasps). We identified $>200$ copies in total, and copy number is dramatically inflated

6 in certain species, such as the spurred ceratina (Ceratina calcarata) which has at

7 least 86 distinct copies (Table 1). The identification of orthologous elements

8 demonstrates these elements derive from a germline incorporation event that

9 occurred >100 Mya (Fig. S4). Furthermore, their occurrence across a wide range of

10 related species suggests that germline incorporation predates deep divergences

11 within the Apoidae, and high copy number reflects subsequent intragenomic

12 amplification. Notably, while most members of this set of elements, labelled EFV-

13 PL.2-Apoidae, are highly degraded, some elements contain relatively intact ORFs,

14 with the longest intact coding regions being identified in C. calcarata - the species

15 with the highest overall copy number (Table 1).

16

\section{Comprehensive phylogenetic analysis revises flavivirus phylogeny}

We performed a comprehensive phylogenetic analysis of the Flaviviridae, using all available information, including endogenous flaviviral elements (EFVs). We used GLUE's 'constrained alignment tree data structure to reconstruct bootstrapped, maximum likelihood phylogenies across a range of taxonomic levels within the Flaviviridae (Table 2, Fig. 2, Fig. S5). Our analysis revealed new information about phylogenetic structure within each major lineage. Within the "pegi-hepaci" lineage the Pegivirus and Hepacivirus genera occupy a derived position [8], viruses identified in sharks group in an intermediate position, and the invertebrate lineage represented by EFV-PegiHepaci-Elysia groups basally (Fig. 2a). The "flavi-pesti" lineage is more structured - it comprises robustly supported "pesti-like" and "flavi-like" clades, each of which has a clear and well-supported internal structure (Fig. 2b). 
In contrast to previous reports [9] we find robust support for a clean division of

2 "flavi-like" viruses into three discrete, monophyletic clades (Fig 2c). Significantly, this

3 reflects a separation between the flaviviruses (genus Flavivirus) and viruses related

4 to 'Tamana bat virus' (TABV), an unusual flavivirus that is often thought of a basal

5 member of genus Flavivirus [23]. However, we identified a Flavivirus-derived EFV in

6 the genome of a basal animal - the freshwater jellyfish Craspadecusta sowerbii - that

7 splits TABV from the Flavivirus genus. This observation, combined with the extensive

8 diversity and broad host of TABV-like viruses, indicates that they represent an

9 entirely distinct subgroup of 'flavi-like' viruses, which we refer to here as genus

10 Tamanavirus, in line with a previous proposal [24] (Fig. 2e).

11 Within the flavivirus genus derived lineages include arthropod-vectored

12 viruses ('arboviruses'), with the tick-borne and mosquito-borne flaviviruses emerging

13 as distinct, robustly supported clades (Fig. 2e, Fig S3b). Additional, basal lineages

14 are present containing viruses with no known vector, and viruses that are restricted

15 to arthropod hosts $[7,9,25]$. In most phylogenies NKV flaviviruses cluster basal to

16 all arthropod/vertebrate flaviviruses, but an alternative topology places these NKV

17 flaviviruses more closely with the tick-borne flaviviruses, as reported by other authors

$18[23,26]$.

19 The remaining "flavi-like" viruses cluster together with jingmenviruses in a

20 robustly supported clade (Fig. 2f). In addition to identifying EFVs that group robustly

21 within the diversity of JMVs, we also identified a more distantly related,

22 'Jingmenvirus-like' lineage - here labelled X2 - that is comprised entirely of EFVs and

23 forms a sister clade to the main JMV lineage (Fig. 2c). Notably, X2-derived EFVs

24 were identified in an arthropod species (the calanoid copepod Eurytemora affinis) as

25 well as in a diverse range of ray-finned fish (clade Actinopterygii) (Table 1). We did

26 not identify any X2-derived insertions that spanned outside of NS5 gene, therefore

27 the event that generated the segmented structure of the JMVs could potentially 
1 predate their split from the $\mathrm{X} 2$ lineage, so that $\mathrm{X} 2$ viruses also have segmented

2 genomes.

The 'pesti-like' lineage contains three robustly supported subclades corresponding to the genus Pestivirus (extended to include basal, pestivirus-like

5 viruses recently identified in lower vertebrates [6]) and two invertebrate-associated

6 clades (here labelled 'PL1' and 'PL2') that have been collectively referred to as 'large

7 genome flaviviruses' (LGFs) [9]. The PL2 subgroup contains both viruses and EFVs

8 (Fig. 2g). The EFV sequences identified in this group are among the largest we

9 identified and included many overlapping fragments that could be assembled into

10 larger genome fragments. However, the longest consensus sequences we could 11 assemble were $\sim 20 \%$ shorter than the genome of the most closely related 12 contemporary virus (Fig. 1b).

\section{All major flavivirus lineages originated in the distant evolutionary past}

We used a variety of approaches to calibrate the evolutionary timeline of the Flaviviridae. Calibrations based on orthology were obtained for jingmenvirus-derived sequences found in the Chironomus midges, for several X2-derived EVEs in fish, and for a PL2-derived EVE in superfamily Apoidea (Table 2). The PL2-derived EFV was the oldest - we identified orthologous loci in two species - the spurred ceratina (Ceratina calcarata) and the green orchid bee (Euglossa dilemma) - that diverged

$21>100$ million years ago (Mya) [27] (Fig. S4). We also derived age estimates ranging between 3-62 Mya for pairs of putatively duplicated EVE sequences based on the assumption a neutral molecular clock (Table 2). show a relatively close evolutionary relationship to contemporary LGFs (Fig 2f). This observation, together with the identification of EFVs in basal animal lineages such as

27 cnidarians and priapulids, suggested to us that the Flaviviridae might have truly 28 primordial origins extending back to the origins of the metazoan $~ 800-500$ Mya. 
1 Consistent with this, we identified several clades in which (i) the branching

2 relationships broadly matched those of host phyla, and (ii) divergence times among

3 animal lineages are significantly correlated with branch lengths (Fig. S6). These

4 included (i) the divergence between invertebrate and vertebrate lineages in the

5 Hepaci-Pegi lineage; (ii) shark/mammal and invertebrate/vertebrate splits in the

6 Pesti-like lineage and (iii) the cnidarian-arthropod, chelicerata-hexapoda, and

7 crustacean-insect splits in the Flavivirus genus.

We integrated the main findings of our comprehensive phylogenetic analysis

9 (Fig. 2) with the various date calibrations obtained via comparative genomic and co-

10

11

12

13

14

15

16

17

18 phyletic analysis to reconstruct a broad overview of Flaviviridae evolution (Fig. 3a) (Table 3). The extended evolutionary timeline implied by our analysis is consistent with a scenario in which vector-borne flaviviruses emerge broadly in parallel with the evolution of hematophagy (blood-feeding) in ticks and insects. We therefore incorporated additional maximum age calibrations based on the assumption that blood-feeding arthropods vectors evolved prior to the emergence of vectored viruses. Similarly, we infer that Gentian Kobu-sho-associated virus (GKaV) - a putative double-stranded RNA virus of plants [10] that groups within the PL1 lineage (Fig 2c) - emerged from an arthropod source via plant feeding (as has been proposed for other enveloped virus groups found in plants [28]), and that both this event and the subsequent transition from single-stranded to double-stranded RNA postdate the evolution and expansion of flowering plants (Angiospermae).

\section{Arthropod-vectored flaviviruses emerged from an arachnid source}

Phylogenetic reconstructions show that the 'classical insect-specific flaviviruses' (cISFs) are robustly separated from the 'classical vector-borne flaviviruses' (CFVs) by viruses identified in crustaceans, sea spiders (Chelicerata), and ticks (Acari) (Fig 3b). In addition, trees rooted on TABV or the cnidarian EFVs show that the most basal CFV lineages are either tick-associated (Mpulungu and the 
1 TBFVs) or potentially tick-associated [23]. In addition, several synapomorphies were

2 identified in the NS5 protein where an ancestral residue is conserved between

3 ancestral and tick-borne flaviviruses, but mutated in insect-vectored flaviviruses (Fig

4 3b, Fig. S7). The most parsimonious interpretation of these observations is that they

5 reflect firstly the emergence of tick-vectored viruses from tick-specific viruses,

6 followed by the emergence of insect-vectored viruses from tick-vectored ancestors

7 on at least one occasion. This model would imply that members of the flavivirus

8 genus for which no known vector has been identified [23] are in reality transmitted by

9 an arthropod vector, or else evolved from viruses that were.

\section{DISCUSSION}

In this study we used EFV sequences to investigate the long-term evolutionary history of the Flaviviridae and calibrate their evolutionary timeline. We identified EFVs derived from a wide range of flavivirus groups in a diverse range of host species. We reveal the first evidence that hepacivirus-like viruses, which have so far only been identified in vertebrates, have counterparts in invertebrate species.

17 We also show that Jingmenvirus-like elements are present in fish, providing first unambiguous evidence for the existence of EFVs in vertebrate genomes. Hepacivirus-related EVEs have previously been reported in rabbits [29], but these sequences are very short (i.e. 20-30 nucleotides) making their origins difficult to 21 investigate further.

We show that flavivirus-like viruses have rarely been fixed in the animal germline - only 36 of 1075 species genomes screened in our analysis contained any EFVs (Table 1). Our screen suggests they most often occur in arthropod genomes, but less commonly than EFVs derived from other RNA virus groups [20]. But while EFVs are rare overall, they occur at relatively high copy number in the germline of 27 some animal species and groups (e.g., Aedes mosquitoes, superfamily Apoidea). 28 High EFV copy number in Aedes species is likely related to role that these 
1 sequences play in antiviral immunity [19]. However, it remains unclear the extent to

2 which it reflects intragenomic duplication of EFVs following rare germline

3 incorporation events, versus numerous distinct incorporations as would be expected

4 for a dynamic system of heritable antiviral immunity [16]. The extremely high copy

5 number of PL2-derived EFVs in superfamily Apoidea could potentially reflect

6 amplification in association with retroelement activity, as has been reported for other

7 kinds of $\operatorname{EVE}[30,31]$, but multiple invasions involving related viruses cannot be ruled

8 out.

The EFVs identified in our study include some remarkably ancient examples

$10>100$ My old, demonstrating that the evolution of the Flaviviridae spans geological

11 eras. Furthermore, we show that calibrations obtained from EVEs can be integrated

12 with dates based on co-phyletic analysis to produce a cohesive overview of flavivirus

13 evolution in which the major flavivirus lineages originated early in animal evolution

14 and broadly co-diverged with animal phyla (Fig 3a). The extended evolutionary

15 timeline implied by our research raises interesting questions about the fundamental

16 way in which animal evolution may have impacted flavivirus evolution, since it

17 encompasses the development of entire organ systems (e.g., the liver and vascular

18 system), and spans the evolution of fundamental ecological shifts such as the

19 emergence of endothermic vertebrates and flowering plants. The identification of

20 flavivirus-derived EVEs in animals lacking a circulatory system (e.g. Cnidarians and

21 Priapulids) is also consistent with a primordial origin in animals, and suggests that

22 virus transmission via exosomes - as has been reported for TBFVs [32] - might

23 represent the most archaic form of cell-to-cell transmission for RNA viruses.

24 The vertebrate blood vascular system evolved $>400$ Mya and was established

25 in its basic form in endothermic vertebrates (birds and mammals) by 200 Mya [33].

26 Once systems for the transport of nutrients had evolved, parasitism of these systems

27 inevitably followed. Accordingly, hematophagous behaviour is thought to have

28 evolved independently in arthropods on >20 occasions [34]. The time-calibrated 
1 phylogeny obtained in our study suggests this also led to independent emergence of

2 arthropod-borne flaviviruses that infect vertebrate species. Besides the various

3 groups of arthropod-borne classical flaviviruses (CFVs), it is now clear that some

$4 \quad \mathrm{JMV}$ are arthropod-borne viruses that infect mammals, including humans [35], and

5 recent studies suggest that dual-host relationships involving vertebrates and

6 arthropods may also exist among tamanaviruses [7]. Furthermore, we find that the

7 JMV-related $\mathrm{X} 2$ lineage is represented by EVEs in both fish and copepod genomes,

8 suggesting it may contain fish viruses that are (or were) transmitted by

9 hematophagous copepods.

10 We show that phylogenetic relationships within the Flavivirus genus are 11 consistent with a scenario under which the ancestors of the arthropod-borne classical 12 flaviviruses (CFVs) circulated exclusively within ancestral arthropods and expanded 13 into vertebrates in association with the evolution of hematophagy in specific 14 arthropod groups (Fig. 2, Fig. 3a). No EVEs derived from the arthropod-borne 15 lineage of classical flaviviruses were identified in our screen and estimates of when 16 hematophagous traits evolved in ticks and mosquitoes vary widely [34]. Thus, we 17 cannot draw definitive conclusions about the timing of their emergence [36]. 18 However, the phylogenetic relationships within genus Flavivirus provide compelling 19 evidence that CFVs originated in arachnids and latterly acquired the capacity to infect 20 and be transmitted by insects (Fig. 3b). This model of CFV evolution has some 21 appealing properties. Firstly, tick feeding entails a relatively long exposure and 22 available evidence indicates that ticks transmit infection to one another via host blood 23 when multiple individuals feed in proximity [37]. To the extent that persisting longer in 24 vertebrate blood is advantageous for increasing transmission among tick populations, 25 it could eventually select for viruses capable of limited replication in vertebrate cells. 26 These viruses could then ultimately evolve into viruses capable of causing sustained 27 viremia, which would in turn create opportunities for biting insects such as 28 mosquitoes to acquire a vector role as they become exposed to tick-vectored viruses 
1 via feeding on viremic vertebrate hosts (Fig. 3c). Interestingly, this 'ticks first'

2 hypothesis is consistent with previous comparative genomics studies, which found

3 evidence that the 3' UTR - which contains numerous RNA structural elements

4 involved in mediating the viral life cycle [38] - evolved via duplications of a 200

5 nucleotide region within the NS5 ORF [39]. These 'long repeated sequences' are

6 best preserved in the TBFV lineage - with only remnants being present in the more

7 derived CFV groups [40].

$8 \quad$ Our findings are consistent with a growing understanding that, despite

9 extremely high background mutation rates and an inherent capacity for rapid

10 adaptation, the overall structural and functional architecture of virus genomes often

11 remains fundamentally conserved over immense expanses of time [30, 41-44]\}. This

12 implies that comparative approaches can be applied to reveal the structural and

13 functional basis of key adaptations, such as the capacity to replicate in both

14 arthropod and vertebrate hosts. In this study we introduced a template for

15 computational genomics studies of viruses that focusses on facilitating the

16 reproduction of comparative analyses and re-use of the complex datasets that

17 underpin them (e.g. MSAs) (Fig. S1). This approach can not only scale to

18 accommodate greatly increased quantities of virus species and sequences, but also

19 introduces new levels of reproducibility and re-usability so that researchers working

20 in different areas of flavivirus genomics - but utilizing related data and domain

21 knowledge - can benefit from one another's work. 


\section{MATERIALS \& METHODS}

\section{Sequences and data}

A library of flavivirus representative genome sequences (Table S1) was collated from GenBank via reference to the International Committee for Taxonomy of

5 Viruses website. Whole genome sequence data of animal species were obtained

6 from the National Center for Biotechnology Information genome database [45]. We

7 obtained all animal genomes available as of March 2020.

Genome screening in silico

WGS data of animal species were obtained from the National Center for

11 Biotechnology Information) genome database [45]. We obtained all animal genomes 12 available as of March 2020. We used the database-integrated genome screening 13 (DIGS) tool [46] to derive a non-redundant database of EFV loci within published 14 WGS assemblies (Table S1). The DIGS tool uses the basic local alignment search 15 tool (BLAST) program suite [47] to perform similarity searches and the MySQL 16 relational database management system (Community Server 8.0.26) to coordinate 17 screening and record output. A user-defined reference sequence library provides 18 both a source of 'probes' for searching WGS data and a means of classifying DNA sequences recovered by screening. We used Flavivirus-GLUE to export a representative library of polypeptide sequences for the Flaviviridae, incorporating all 21 previously characterised flavivirus species and EVEs, as input for DIGS (Fig S8). 22 Sequences that could produce false positive matches - e.g. the host-derived 23 sequence insertions found in the reference genome sequence of bovine viral 24 diarrhoea virus 1 [48] - were excluded from the probe set. We extended the 25 screening database schema to incorporate tables representing the taxonomic 26 classifications of viruses, EFVs and host species, and used database queries 27 constructed using SQL to filter matches based on their similarity reference 28 sequences and their taxonomic distribution across hosts. Using this approach, we 
1 categorised sequences into: (i) putatively novel EFV elements; (ii) orthologs of

2 previously characterised EFVs (e.g., copies containing large indels); (iii) non-viral

3 sequences that cross-matched to flavivirus probes (e.g., retrotransposons). For

4 genome sequences that were obtained from cell lines we discarded two sequences

5 that disclosed $<3 \%$ nucleotide divergence from contemporary Dengue strains, as

6 these could potentially represent integrations that occurred in cell culture.

$7 \quad$ EFVs were assigned standard identifiers following a convention established

8 for endogenous retroviruses [49] - the first component is the classifier 'EFV' (which

9 may be dropped when it is implied by context). The second component comprises

10 two subcomponents separated by a period; (i) the name of the taxonomic group of

11 viruses from which the EFV is thought to derive (the lowest level taxonomic group

12 into which the element can be confidently placed by phylogenetic analysis is used);

13 (ii) a numeric ID that uniquely identifies the integration event from which the insertion

14 derives within the taxonomic lineage it is assigned to. The third component describes

15 the known taxonomic distribution of orthologous copies of the element among host

16 species. For the Jingmenviruses, which have segmented genomes, we used

17 classifiers that specify the gene from which the EVE is derived, in line with

18 conventions established for EVEs derived from mRNAs and from segmented viruses

19 [43]- endogenous jingmenvirus helicase (EJH) and endogenous jingmenvirus

20 polymerase (EJP).

$\underline{\text { Phylogenetic analysis }}$

A process for reconstructing evolutionary relationships across the entire

24 Flaviviridae was implemented using GLUE. We used a data structure called a 25 'constrained MSA tree' to coordinate genomic analyses across the large phylogenetic 26 distances found in flaviviruses Fig. S7d. This approach addresses the issue that 27 MSAs constructed at higher taxonomic levels (e.g., above genus-level in the 28 Flaviviridae) typically contain far fewer columns than those constructed at low 
1 taxonomic levels (e.g., subgenus), meaning that to fully investigate phylogenetic

2 relationships using all of the available information it is often necessary to construct

3 several separate MSAs each representing a distinct taxonomic grouping (e.g. see

4 Table 2). The difficulties encountered in maintaining these MSAs in sync with one

5 another while avoiding irreversible data loss are a key factor underlying the low levels

6 of reproducibility and re-use in comparative genomic analyses [50], particularly those

7 that examine more distant evolutionary relationships genomic [51]. To address these

8 issues the 'constrained MSA tree' data structure represents the hierarchical links

9 between MSAs constructed at distinct taxonomic levels, creating in effect a single,

10 unified MSA that can be used to reconstruct both shallow and deep evolutionary

11 relationships while making use of the maximum amount of available information at

12 each level (Table 2, Fig. S1d). This approach also has the effect of standardising the

13 genomic coordinate space to the constraining reference sequences selected for each

14 MSA without imposing any limitations on which references are used (e.g., laboratory

15 strains versus wild-type references), since additional, alternative constraining

16 references can be incorporated, and contingencies such as insertions relative to the

17 constraining reference are dealt with in a standardised way [21].

18 MSAs partitions derived from the constrained MSA tree were used as input

19 for phylogenetic reconstructions. Nucleotide and protein phylogenies were

20 reconstructed using maximum likelihood (ML) as implemented in RAxML (version

21 8.2.12) [52]. To handle coverage-related issues we generated gene coverage data

22 prior to phylogenetic analysis and used this information to condition the way in which

23 taxa are selected into MSA partitions (Supplementary). Protein substitution models

24 were selected via hierarchical maximum likelihood ratio test using the

25 PROTAUTOGAMMA option in RAXML.

26

$27 \quad$ Genomic analysis 
bioRxiv preprint doi: https://doi.org/10.1101/2021.09.19.460981; this version posted September 21, 2021. The copyright holder for this preprint (which was not certified by peer review) is the author/funder, who has granted bioRxiv a license to display the preprint in perpetuity. It is made available under aCC-BY-NC-ND 4.0 International license.

1

Open reading frames (ORFs) of EVEs were inferred by manual comparison to

2 virus genomes. We used a BLAST-based, codon-aware aligner implemented in

3 GLUE [21, 47] to generate in-frame alignments of virus and EFV virus coding

4 domains, and to assemble a consensus genome of sea spider flavivirus from

5 published transcripts [53]. JalView [54] (version 2.11.1.4) and Se-Al (version 2.0a11)

6 
1 Figure 1. Genome structures of endogenous flaviviral elements.

2 Schematic diagrams showing the genomic regions represented by endogenous

3 flaviviral element (EFV) sequences. (a) Hepaci-Pegivirus-like elements are shown

4 relative to hepatitis $\mathrm{C}$ virus (HCV); (b) elements derived from the 'Pestivirus-like 2'

5 (PL2) lineage of viruses shown relative to Shuangao lacewing virus 2 (SLwV-2); (c)

6 Elements derived from the Flavivirus genus, the proposed 'Tamanavirus' genus, and

7 the X1 lineage shown relative to Dengue virus type 1 (DENV-1); (d) Jingmenvirus-

8 derived EFVs shown relative to Jingmen tick virus (JMTV). Homologous regions

9 represented by EFV sequences are shown as grey horizontal bars. Bars to the right

10 show taxonomic groups. EFV identifiers (IDs) are shown to the left. EFV IDs were

11 constructed as indicated in the key, following a convention established for

12 endogenous retroviruses [49]. Abbreviations: $\mathrm{Kb}=$ kilobase. $\mathrm{X} 1=$ unclassified

13 flavivirus-like lineage X1. X2 = unclassified flavivirus-like lineage X2. C = Capsid, prM

$14=$ Pre-Membrane, $\mathrm{E}=$ Envelope, $\mathrm{NS}=$ Non-Structural protein, $\mathrm{P}=$ Protease, $\mathrm{H}=$ 15 Helicase. HP = Hepacivirus/Pegivirus-like virus. * Indicates consensus sequence.

16

17

18

22

23

24

25

26

27

28

Figure 2. Phylogenetic relationships of Flaviviruses and endogenous flaviviral elements (EFVs). Bootstrapped maximum likelihood phylogenies (1000 replicates), reconstructed for viruses and EFVs across a range of taxonomic levels, as follows: (a) All Flaviviridae, showing placement of Hepacivirus-related EFV (122 amino acid (aa) residues in multiple sequence alignment (MSA) spanning conserved regions in NS3, substitution model=RTREV); (b) Flavi-Pesti lineage (MSA spanning 99 aa residues in NS5, substitution model= BLOSUM62); (c) Pesti-like lineage (104 aa residues in NS5, substitution model=RTREV); (d) Flavi-like lineage (MSA spanning 179 aa residues in NS5, substitution model=LG likelihood); (e) Tentative genus 'Tamanavirus' (MSA spanning 292 aa residues in NS5, substitution model=BLOSUM62); (f) Jingmenviruses and related lineages (MSA spanning 727 
1 residues in NS5, substitution model=LG likelihood). EFV names are shown in bold.

2 Only EFVs that had sufficient coverage ( $>50 \%$ of total MSA length) were included in

3 the analysis. Triangular terminal branches indicate collapsed clades containing

4 multiple taxa. Asterisks indicate bootstrap support $\geq 70 \%$ (1000 replicates). The scale

5 bar indicates evolutionary distance in substitutions per site. Brackets to the right

6 indicate genera and sub-lineages. All trees are midpoint rooted for display purposes.

7 Abbreviations: cISF $=$ classical insect-specific flaviviruses. NKV $=$ no known vector.

$8 \quad$ Jingmen $=$ Jingmen tick virus $=. \mathrm{PL}=$ Pestivirus-like; MBFV2 = Mosquito borne.

9

Figure 3. Timeline of flavivirus evolution

11

12

13

14

15

16

17

18

19

20

21

22

23

24

25

26

27

28

(a) An integrated overview of flavivirus evolution. A time-calibrated phylogeny, summarising the phylogenetic relationships recovered in our analysis is shown. The key indicates the different types of time calibrations incorporated into the figure. Numbers adjacent to key symbols correspond to numbered rows in Table $\mathbf{3}$ 'Codivergence type $A^{\prime}$ = codivergence supported by co-phylogeny. Codivergence type $B=$ potential codivergence-based calibrations without supporting evidence. Vertical lines to right of the tree show taxonomic groups.

(b) Evolution of arthropod-borne flaviviruses. The phylogeny shown on the left was constructed from an alignment spanning 804 residues of the precursor polyprotein (substitution model=LG likelihood). The phylogeny on the right is a timecalibrated phylogeny of arthropod hosts/vectors of classical flaviviruses, obtained via TimeTree [27]. The figure shows the hypothesised relationships between the host and virus trees, with virus phylogeny broadly following host phylogeny at higher taxonomic levels but diverging dramatically from this pattern when tick-borne viruses of vertebrates emerge. 
1 (c) Model for the origin and evolution of the vector-borne flaviviruses. Three

2 stages are shown: (i) an ancestral group of non-vectored tick viruses is present; (ii)

3 tick hematophagy provides prolonged exposure to vertebrate blood allowing tick-

4 specific flaviviruses to eventually acquire the ability to replicate in vertebrate cells and

5 become tick-borne flaviviruses (TBFV); (iii) the evolution of TBFV gives rise to the

6 occurrence of viremic vertebrate hosts, thereby exposing mosquitoes and other

7 hematophagous insects such that they might acquire a role as vectors.

8 
bioRxiv preprint doi: https://doi.org/10.1101/2021.09.19.460981; this version posted September 21, 2021. The copyright holder for this preprint (which was not certified by peer review) is the author/funder, who has granted bioRxiv a license to display the preprint in perpetuity. It is made available under aCC-BY-NC-ND 4.0 International license.

Table 1. Endogenous flaviviral elements detected in this study

\begin{tabular}{|c|c|c|c|c|c|}
\hline Sequence ID $^{a}$ & \#Species $^{b}$ & \#Sequences $^{c}$ & IORF $^{d}$ & Host species $^{e}$ & Common name \\
\hline \multicolumn{6}{|l|}{ Flavivirus } \\
\hline clSF.1-AedAeg ${ }^{*}$ & 1 & 92 & 564 & Aedes aegypti & Yellow fever mosquito \\
\hline cISF.2-AedAlb* & 1 & 35 & 643 & Aedes albopictus & Tiger mosquito \\
\hline cISF.3-AnoMin* & 1 & 1 & 97 & Anopheles minimus & \\
\hline clSF.4-AnoSin* & 1 & 1 & 138 & Anopheles sinensis & \\
\hline cISF.5-TipOle & 1 & 4 & 226 & Tipula oleracea & Marsh crane fly \\
\hline cISF.6-ConPat & 1 & 1 & 38 & Condylostylus patibulatus & Long-legged fly \\
\hline Flavi.1-CraSow & 1 & 13 & 1182 & Craspedacusta sowerbyi & Freshwater jellyfish \\
\hline Flavi.2-DapMag* & 1 & 2 & 573 & Daphnia magna & Water flea \\
\hline Flavi.3-LepArc* & 1 & 3 & 281 & Lepidurus arcticus & Tadpole shrimp \\
\hline \multicolumn{6}{|l|}{ Tamanavirus } \\
\hline Tamana.1-LedTum & 1 & 1 & 55 & Lednia tumana & Meltwater stonefly \\
\hline Tamana.2-LauKoh & 1 & 1 & 56 & Laupala kohalensis & Hawaiian cricket \\
\hline Tamana.3-AmpSul & 1 & 2 & 44 & Amphinemura sulcicollis & Spring stonefly \\
\hline Tamana.4-StyCho & 1 & 1 & 873 & Stylephorus chordatus & Tube-eye \\
\hline \multicolumn{6}{|l|}{ Jingmenvirus } \\
\hline $\begin{array}{l}\text { EJP-Jingmen.1- } \\
\text { Chironomus }\end{array}$ & 2 & 2 & 248 & Chironomus sp. & \\
\hline EJP-Jingmen.2-Gerris & 1 & 1 & 45 & Gerris buenoi & Water strider \\
\hline EJH-Jingmen.1-Gerris & 1 & 1 & 51 & “ & \\
\hline \multicolumn{6}{|l|}{$\mathbf{x} 1$} \\
\hline X1.1-PriCau & 1 & 2 & 347 & Priapulus caudatus & Penis worm \\
\hline \multicolumn{6}{|l|}{$\mathrm{X} 2$} \\
\hline X2.1-AusLim & 1 & 2 & 214 & Austrofundulus limnaeus & Mangrove killifish \\
\hline X2.2-StyCho & 1 & 3 & 873 & Stylephorus chordatus & Tube-eye \\
\hline X2.3-EurAff & 1 & 2 & 190 & Eurytemora affinis & Copepod \\
\hline X2.4-Takifugu & 3 & 5 & 103 & Takifugu & Pufferfish \\
\hline X2.5-Phycis & 2 & 2 & 129 & Phycis & Phycid hakes \\
\hline X2.6-MorMor & 1 & 1 & 36 & Mora moro & Common mora \\
\hline X2.7-BroBro & 1 & 1 & 56 & Brosme brosme & Cusk \\
\hline X2.8-MacPee & 1 & 1 & 51 & Maccullochella peelii & Murray cod \\
\hline X2.9-BolPec & 1 & 1 & 155 & $\begin{array}{l}\text { Boleophthalmus } \\
\text { pectinirostris }\end{array}$ & $\begin{array}{l}\text { Bluespotted } \\
\text { mudhopper }\end{array}$ \\
\hline \multicolumn{6}{|l|}{ Pesti-like } \\
\hline PL2.1-CalCec & 1 & 5 & 78 & Calycopis cecrops & $\begin{array}{l}\text { Red-banded } \\
\text { hairstreak }\end{array}$ \\
\hline PL2.2-EucHer & 1 & 2 & 187 & Eucheros histo & Stink bug \\
\hline PL2.3-Apoidae & 11 & 239 & 2469 & Family Apoidae & Sweat bees \\
\hline PL2.4-AndCur & 2 & 6 & 961 & Andricus & Cynipid gall wasp \\
\hline PL2.5-AnoGla & 1 & 1 & 26 & Anoplophora glabripennis & Long-horned beetle \\
\hline PL2.6-XenBra & 1 & 16 & 2232 & Xenocatantops brachycerus & Grasshopper \\
\hline PL2.7-OpeBru & 1 & 2 & 38 & Operophtera brumata & Winter moth \\
\hline \multicolumn{6}{|l|}{ Pegi-Hepaci } \\
\hline HepaPegi.1-ElyChl & 1 & 1 & 60 & Elysia chlorotica & $\begin{array}{l}\text { Eastern emerald } \\
\text { elysia }\end{array}$ \\
\hline
\end{tabular}

Footnote: ${ }^{a} \mathrm{EFV}$ s have been assigned standard IDs based on conventions established for endogenous retroviruses, wherein information about virus taxonomy and locus orthology are incorporated into the ID itself (see Methods). Asterisks indicate EFVs loci or lineages that have been reported previously. For jingmenviruses, the classifier component of the ID specifies the gene it is derived from following conventions established for EFVs derived from

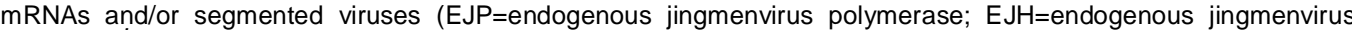
helicase). ${ }^{b}$ Number of species in which EVE locus was identified. ${ }^{c}$ Number of sequences (i.e., distinct insertions) derived from this EVE that were identified via in silico screening. ${ }^{e}$ Host species or species groups. Abbreviations: $L-$ $\mathrm{ORF}=$ Longest open reading frame; PL2=Pesti-like 2; clSF=classical insect-specific flaviviruses. 
Table 2. Comprehensive mapping of flavivirus homology via hierarchically linked multiple sequence alignments

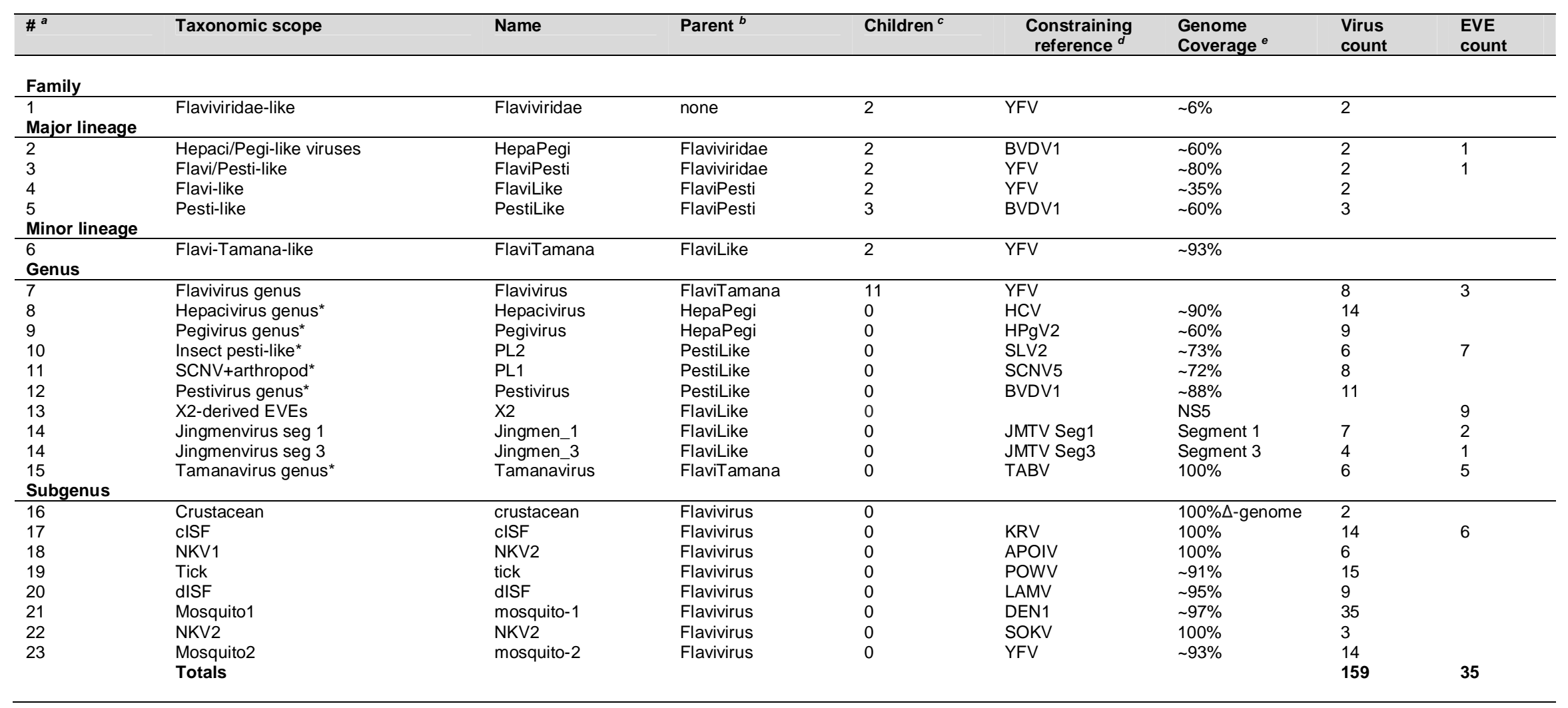

Footnote: ${ }^{a}$ Numbers correspond to labelled nodes in Fig. S1d. ${ }^{b}$ The parent multiple sequence alignment (MSA) in the hierarchy. ${ }^{c}$ Children of the MSA in the hierarchy. ${ }^{d}$ Reference sequence that constrains the genomic co-ordinate coordinate space in the MSA. ${ }^{e}$ Percentage of the constraining reference genome spanned by the MSA. 
bioRxiv preprint doi: https://doi.org/10.1101/2021.09.19.460981; this version posted September 21, 2021. The copyright holder for this preprint (which was not certified by peer review) is the author/funder, who has granted bioRxiv a license to display the preprint in perpetuity. It is made available under aCC-BY-NC-ND 4.0 International license.

Table 3. Dates and age estimates used to calibrate flavivirus evolution.

\begin{tabular}{|c|c|c|c|c|c|}
\hline \# & Virus lineage & Host lineage(s) & MYA & Low & High \\
\hline \multicolumn{6}{|c|}{ Codivergence $\mathbf{A}$ (minimum age) } \\
\hline 1 & Flaviviridae & Animalia & 952 & 757 & 1147 \\
\hline 6 & Jingmen-X2 & Insecta-Crustacea & 794 & 678 & 916 \\
\hline 22 & Mosquito 2 subgenus & Culex-Aedes & 40 & 22 & 52 \\
\hline 10 & Jingmenvirus & Arachnida-Insecta & 601 & 568 & 642 \\
\hline \multicolumn{6}{|c|}{ Codivergence B (minimum age) } \\
\hline 2 & Hepaci-Pegi lineage & Mollusc-vertebrate & 794 & 678 & 916 \\
\hline 3 & Pesti-like viruses & Invertebrates-vertebrates & 794 & 678 & 916 \\
\hline 4 & Pegi-Hepaci & Chondrichthyes-Mammalia & 465 & 450 & 497 \\
\hline 5 & Pestiviruses & Chondrichthyes-Mammalia & 465 & 450 & 497 \\
\hline 7 & Flavivirus & Cnidaria-Arthropoda & 824 & 652 & 973 \\
\hline 8 & Arthropod flaviviruses & Crustacea-Arachnida & 601 & 568 & 642 \\
\hline 9 & Chelicerata flaviviruses & Pycnogonida-Arachnida & 553 & 476 & 653 \\
\hline & & & & & \\
\hline \multicolumn{6}{|c|}{ Orthology (minimum age) } \\
\hline 13 & PL2 & Family Apoidea & 102 & 71 & 148 \\
\hline 24 & Jingmenvirus & Genus Chironomus & $n / k$ & $n / k$ & $n / k$ \\
\hline 23 & $\mathrm{X} 2$ & Genus Phycis & 16.9 & 12.1 & 37.1 \\
\hline \multicolumn{6}{|c|}{ Duplicates /Molecular clock (minimum age) } \\
\hline 20 & cISF & Tipula & & 16 & 40 \\
\hline 21 & Crustacean FV & Daphnia 2 & & 26 & 62 \\
\hline 19 & Cnidarian FV & Craspedacusta 1 & & 17 & 40 \\
\hline 16 & $\mathrm{X} 1$ & Priapulus (NS5) & & 12 & 29 \\
\hline 17 & $\mathrm{X} 2$ & Eurytemora (NS5) & & 20 & 47 \\
\hline 18 & Tamanavirus & Austrofundulus 1 (NS5) & & 19 & 43 \\
\hline 14 & PL2 & Operophtera & & 17 & 40 \\
\hline \multicolumn{6}{|c|}{ Origin hypothesis (maximum age) } \\
\hline 11 & All vectored flaviviruses & Tick hematophagy & 300 & 90 & 400 \\
\hline 12 & Mosquito-vectored flaviviruses & Mosquito hematophagy & & 79 & 100 \\
\hline 15 & Gentian Kobu-Sho virus & Evolution of angiosperms & & 190 & 315 \\
\hline
\end{tabular}

Footnote: Node numbers correspond to those shown in Fig. 3a. Hematophagy estimates obtained from [34]. Culex-Aedes divergence date obtained from [55]. Angiosperm emergence taken from [56]. All other divergence data estimates were obtained from TimeTree [27]. 


\section{Acknowledgements}

RJG was funded by the Medical Research Council of the United Kingdom (MC_UU_12014/12). We thank Joseph Hughes, Alain Kohl, Spyros Lytras, Emilie Pondeville, Charles Rice, David Robertson, Greg Towers, and Sam J. Wilson for critical reading of the manuscript.

\section{References}

1. Manns, M.P., et al., Hepatitis C virus infection. Nature Reviews Disease Primers, 2017. 3(1): p. 17006.

2. Pierson, T.C. and M.S. Diamond, The continued threat of emerging flaviviruses. Nat Microbiol, 2020. 5(6): p. 796-812.

3. Simmonds, P., et al., ICTV Virus Taxonomy Profile: Flaviviridae. J Gen Virol, 2017. 98(1): p. 2-3.

4. Chambers, T.J., et al., Flavivirus genome organization, expression, and replication. Annu Rev Microbiol, 1990. 44: p. 649-88.

5. Shi, M., et al., Divergent Viruses Discovered in Arthropods and Vertebrates Revise the Evolutionary History of the Flaviviridae and Related Viruses. J Virol, 2016. 90(2): p. 659-69.

6. Shi, M., et al., The evolutionary history of vertebrate RNA viruses. Nature, 2018. 556(7700): p. 197-202.

7. Parry, R. and S. Asgari, Discovery of novel crustacean and cephalopod flaviviruses: insights into evolution and circulation of flaviviruses between marine invertebrate and vertebrate hosts. J Virol, 2019.

8. Porter, A.F., et al., Novel hepaci- and pegi-like viruses in native Australian wildlife and non-human primates. Virus Evol, 2020. 6(2): p. veaa064.

9. Paraskevopoulou, S., et al., Viromics of extant insect orders unveil the evolution of the flavi-like superfamily. Virus Evol, 2021. 7(1): p. veab030.

10. Kobayashi, K., et al., Gentian Kobu-sho-associated virus: a tentative, novel double-stranded RNA virus that is relevant to gentian Kobu-sho syndrome. Journal of General Plant Pathology, 2012. 79: p. 56-63.

11. Qin, X.C., et al., A tick-borne segmented RNA virus contains genome segments derived from unsegmented viral ancestors. Proc Natl Acad Sci U S A, 2014. 111(18): p. 6744-9.

12. Gould, E.A., et al., Origins, evolution, and vector/host coadaptations within the genus Flavivirus. Adv Virus Res, 2003. 59: p. 277-314.

13. Duchene, S., E.C. Holmes, and S.Y. Ho, Analyses of evolutionary dynamics in viruses are hindered by a time-dependent bias in rate estimates. Proc Biol Sci, 2014. 281(1786).

14. Aiewsakun, P. and A. Katzourakis, Time-Dependent Rate Phenomenon in Viruses. J Virol, 2016. 90(16): p. 7184-95.

15. Katzourakis, A. and R.J. Gifford, Endogenous viral elements in animal genomes. PLoS Genet, 2010. 6(11): p. e1001191.

16. Ophinni, Y., et al., piRNA-Guided CRISPR-like Immunity in Eukaryotes. Trends Immunol, 2019. 40(11): p. 998-1010.

17. Lequime, S. and L. Lambrechts, Discovery of flavivirus-derived endogenous viral elements in Anopheles mosquito genomes supports the existence of Anopheles-associated insect-specific flaviviruses. Virus Evol, 2017. 3(1): p. vew035.

18. Whitfield, Z.J., et al., The Diversity, Structure, and Function of Heritable Adaptive Immunity Sequences in the Aedes aegypti Genome. Curr Biol, 2017. 27(22): p. 3511-3519.e7. 
19. Suzuki, Y., et al., Non-retroviral Endogenous Viral Element Limits Cognate Virus Replication in Aedes aegypti Ovaries. Curr Biol, 2020. 30(18): p. 34953506.e6.

20. Blair, C.D., K.E. Olson, and M. Bonizzoni, The Widespread Occurrence and Potential Biological Roles of Endogenous Viral Elements in Insect Genomes. Curr Issues Mol Biol, 2020. 34: p. 13-30.

21. Singer, J.B., et al., GLUE: a flexible software system for virus sequence data. BMC Bioinformatics, 2018. 19(1): p. 532.

22. Merkel, D., Docker: lightweight linux containers for consistent development and deployment. Linux Journal, 2014. 239(2).

23. Blitvich, B.J. and A.E. Firth, A Review of Flaviviruses that Have No Known Arthropod Vector. Viruses, 2017. 9(6).

24. de Lamballerie, X., et al., Genome sequence analysis of Tamana bat virus and its relationship with the genus Flavivirus. J Gen Virol, 2002. 83(Pt 10): p. 2443-54.

25. Blitvich, B.J. and A.E. Firth, Insect-specific flaviviruses: a systematic review of their discovery, host range, mode of transmission, superinfection exclusion potential and genomic organization. Viruses, 2015. 7(4): p. 1927-59.

26. Temmam, S., et al., Characterization of Viral Communities of Biting Midges and Identification of Novel Thogotovirus Species and Rhabdovirus Genus. Viruses, 2016. 8(3): p. 77.

27. Kumar, S., et al., TimeTree: A Resource for Timelines, Timetrees, and Divergence Times. Mol Biol Evol, 2017. 34(7): p. 1812-1819.

28. Buchmann, J.P. and E.C. Holmes, Cell Walls and the Convergent Evolution of the Viral Envelope. Microbiol Mol Biol Rev, 2015. 79(4): p. 403-18.

29. Silva, E., et al., Endogenous hepatitis $C$ virus homolog fragments in European rabbit and hare genomes replicate in cell culture. PLoS One, 2012. 7(11): p. e49820.

30. Lytras, S., G. Arriagada, and R.J. Gifford, Ancient evolution of hepadnaviral paleoviruses and their impact on host genomes. Virus Evol, 2021. 7(1): p. veab012.

31. Inoue, Y., et al., Complete fusion of a transposon and herpesvirus created the Teratorn mobile element in medaka fish. Nat Commun, 2017. 8(1): p. 551.

32. Zhou, W., et al., Exosomes serve as novel modes of tick-borne flavivirus transmission from arthropod to human cells and facilitates dissemination of viral RNA and proteins to the vertebrate neuronal cells. PLoS Pathog, 2018. 14(1): p. e1006764.

33. Monahan-Earley, R., A.M. Dvorak, and W.C. Aird, Evolutionary origins of the blood vascular system and endothelium. J Thromb Haemost, 2013. 11 Suppl 1(Suppl 1): p. 46-66.

34. Mans, B.J., Evolution of vertebrate hemostatic and inflammatory control mechanisms in blood-feeding arthropods. J Innate Immun, 2011. 3(1): p. 4151.

35. Wang, Z.D., et al., A New Segmented Virus Associated with Human Febrile Illness in China. N Engl J Med, 2019. 380(22): p. 2116-2125.

36. Mans, B.J., A.I. Louw, and A.W.H. Neitz, Evolution of Hematophagy in Ticks: Common Origins for Blood Coagulation and Platelet Aggregation Inhibitors from Soft Ticks of the Genus Ornithodoros. Molecular Biology and Evolution, 2002. 19(10): p. 1695-1705.

37. Hermance, M.E. and S. Thangamani, Tick $\square$ Virus $\square$ Host Interactions at the Cutaneous Interface: The Nidus of Flavivirus Transmission. Viruses, 2018. 10(7).

38. Ochsenreiter, R., I.L. Hofacker, and M.T. Wolfinger, Functional RNA Structures in the 3'UTR of Tick-Borne, Insect-Specific and No-Known-Vector Flaviviruses. Viruses, 2019. 11(3). 
39. Gritsun, T.S. and E.A. Gould, Direct repeats in the flavivirus 3' untranslated region; a strategy for survival in the environment? Virology, 2007. 358(2): $p$. 258-65.

40. Gritsun, D.J., et al., Molecular archaeology of Flaviviridae untranslated regions: duplicated RNA structures in the replication enhancer of flaviviruses and pestiviruses emerged via convergent evolution. PLoS One, 2014. 9(3): p. e92056.

41. Gifford, R.J., Viral evolution in deep time: lentiviruses and mammals. Trends Genet, 2012. 28(2): p. 89-100.

42. Zhang, Y.Z., et al., The diversity, evolution and origins of vertebrate RNA viruses. Curr Opin Virol, 2018. 31: p. 9-16.

43. Kawasaki, J., et al., 100-My history of bornavirus infections hidden in vertebrate genomes. Proc Natl Acad Sci U S A, 2021. 118(20).

44. Barreat, J.G.N. and A. Katzourakis, Paleovirology of the DNA viruses of eukaryotes. Trends Microbiol, 2021.

45. Kitts, P.A., et al., Assembly: a resource for assembled genomes at NCBI. Nucleic Acids Res, 2016. 44(D1): p. D73-80.

46. Zhu, H., et al., Database-integrated genome screening (DIGS): exploring genomes heuristically using sequence similarity search tools and a relational database. bioRxiv, 2018.

47. Altschul, S.F., et al., Gapped BLAST and PSI-BLAST: a new generation of protein database search programs. Nuc. Acids Res., 1997. 25: p. 3389-3402.

48. Rinck, G., et al., A cellular J-domain protein modulates polyprotein processing and cytopathogenicity of a pestivirus. J Virol, 2001. 75(19): p. 9470-82.

49. Gifford, R.J., et al., Nomenclature for endogenous retrovirus (ERV) loci. Retrovirology, 2018. 15(1): p. 59.

50. Grüning, B., et al., Practical Computational Reproducibility in the Life Sciences. Cell Syst, 2018. 6(6): p. 631-635.

51. Holmes, E.C. and S. Duchêne, Can Sequence Phylogenies Safely Infer the Origin of the Global Virome? mBio, 2019. 10(2).

52. Stamatakis, A., RAxML version 8: a tool for phylogenetic analysis and postanalysis of large phylogenies. Bioinformatics, 2014. 30(9): p. 1312-3.

53. Conway, M.J., Identification of a Flavivirus Sequence in a Marine Arthropod. PLoS One, 2015. 10(12): p. e0146037.

54. Waterhouse, A.M., et al., Jalview Version 2--a multiple sequence alignment editor and analysis workbench. Bioinformatics, 2009. 25(9): p. 1189-91.

55. Sieglaff, D.H., et al., Comparative genomics allows the discovery of cisregulatory elements in mosquitoes. Proc Natl Acad Sci U S A, 2009. 106(9): p. 3053-8.

56. Qin, L., et al., Insights into angiosperm evolution, floral development and chemical biosynthesis from the Aristolochia fimbriata genome. Nat Plants, 2021. 


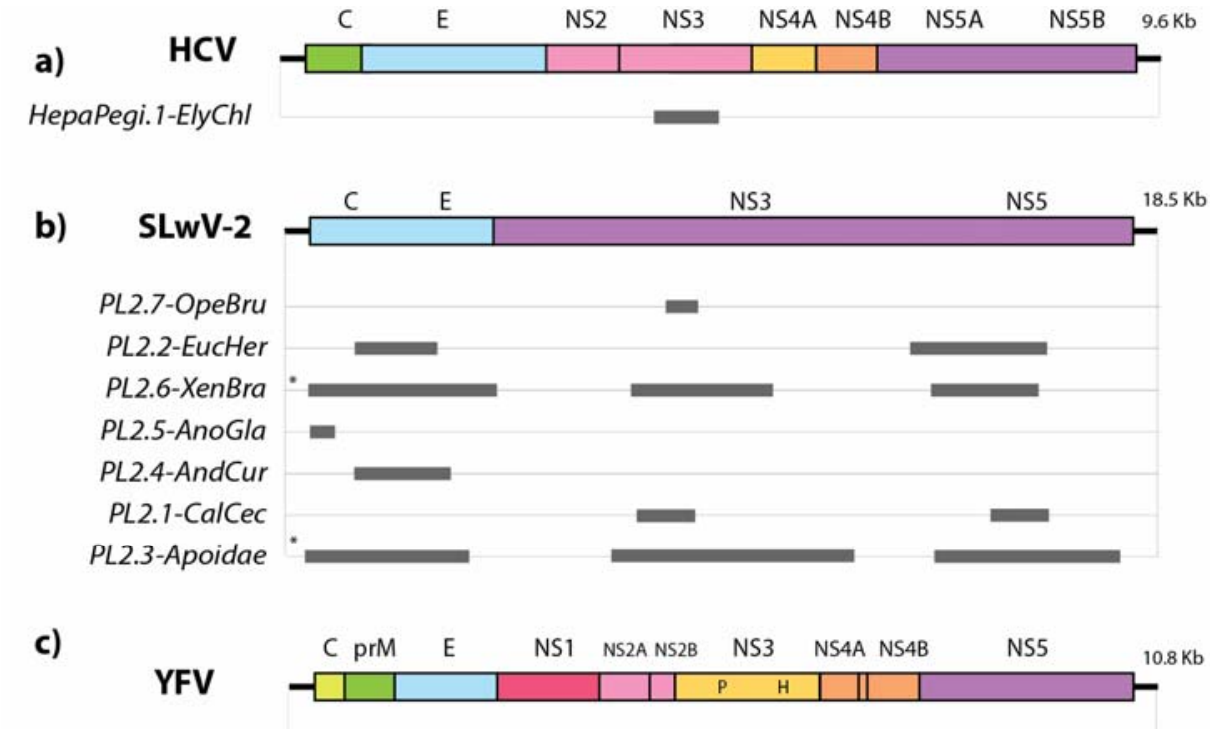

d)
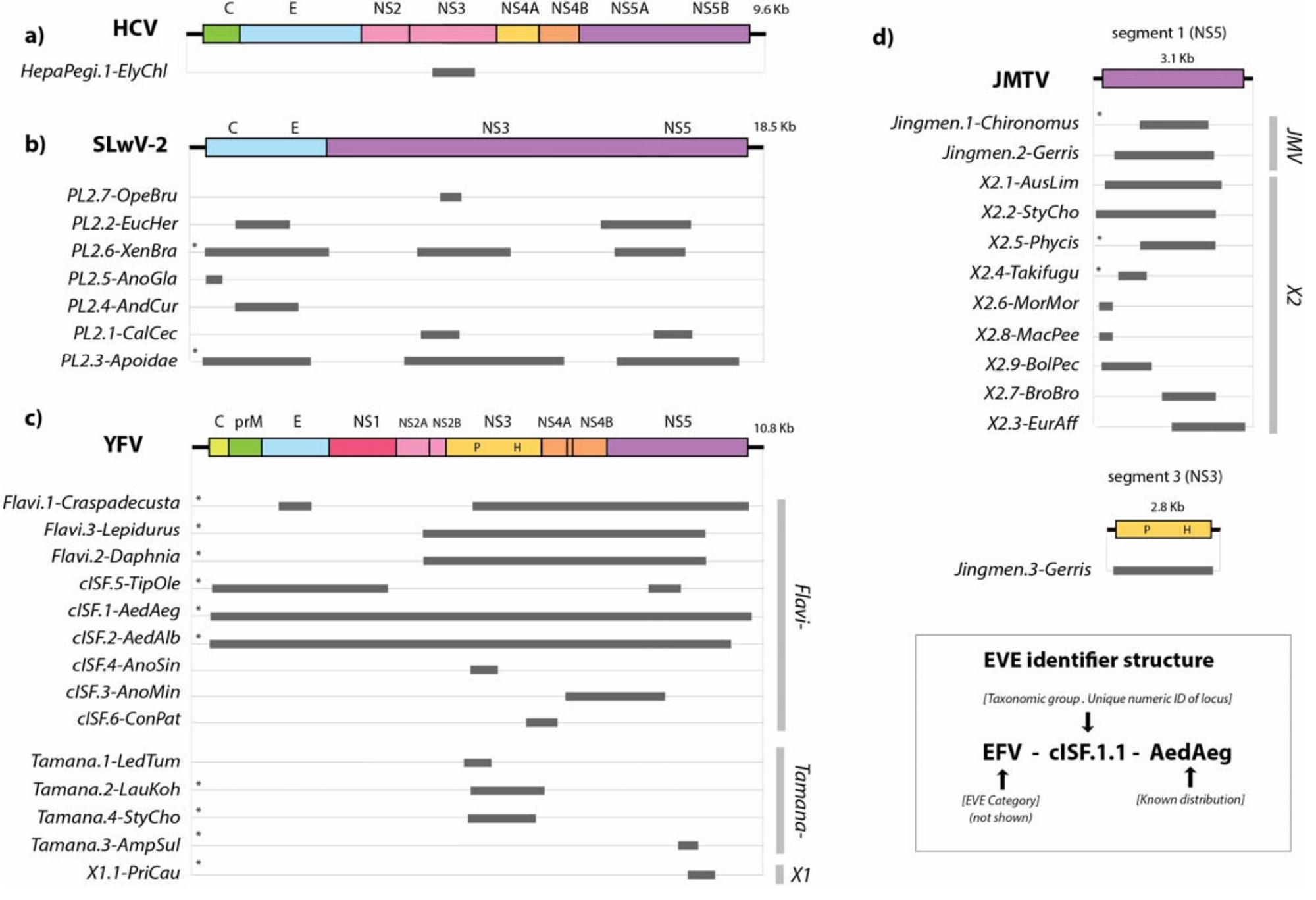
a)

\section{Flaviviridae}

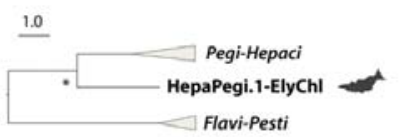

b)

Flavi-Pesti

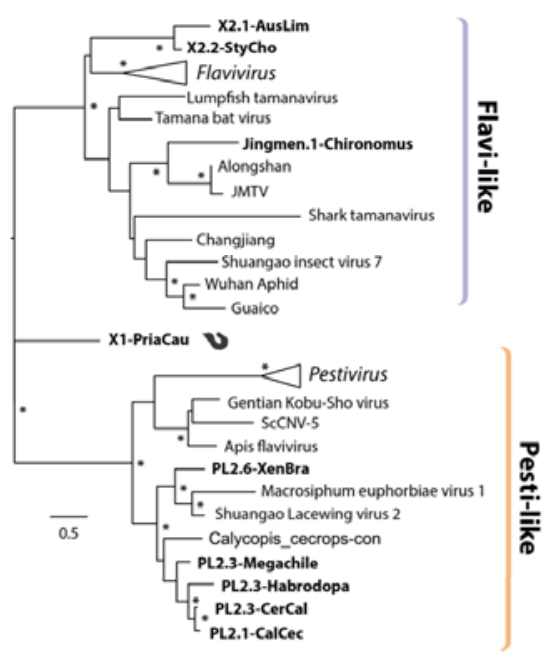

* Ticks (Acari)

$T$ Copepods c)
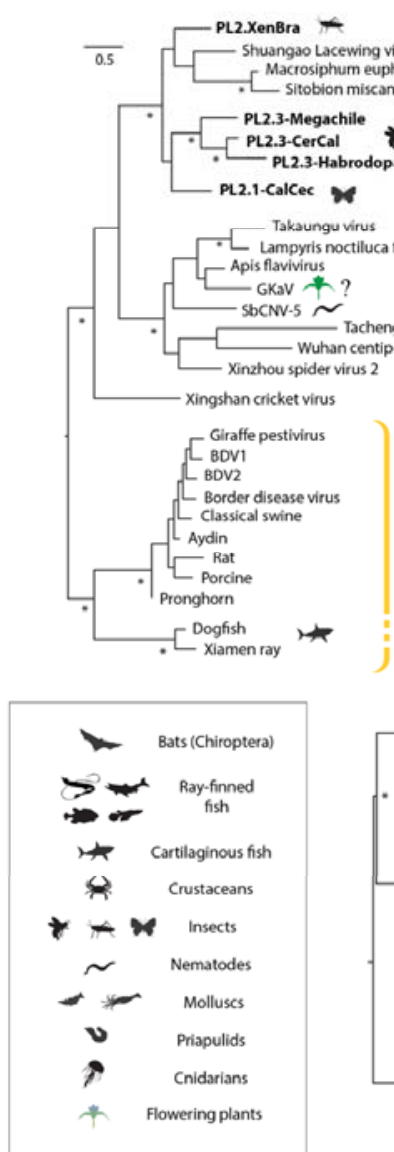

Pesti-like

e
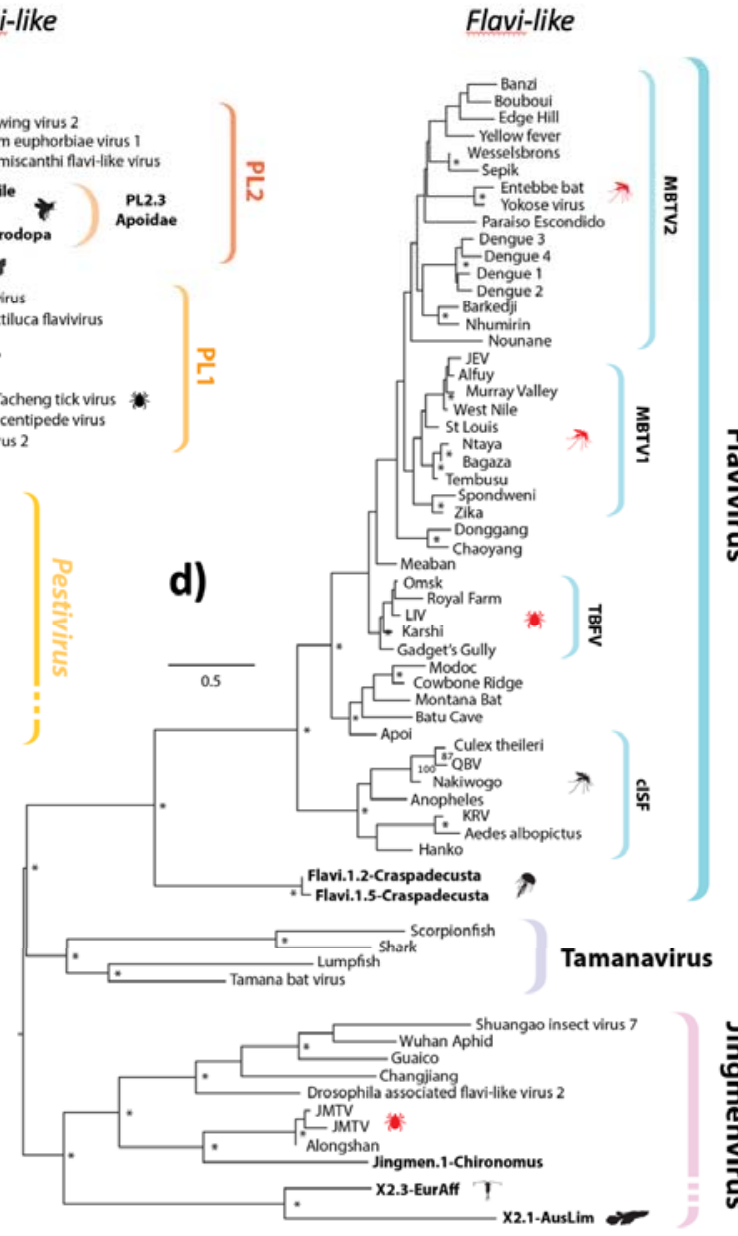

Flavi-like
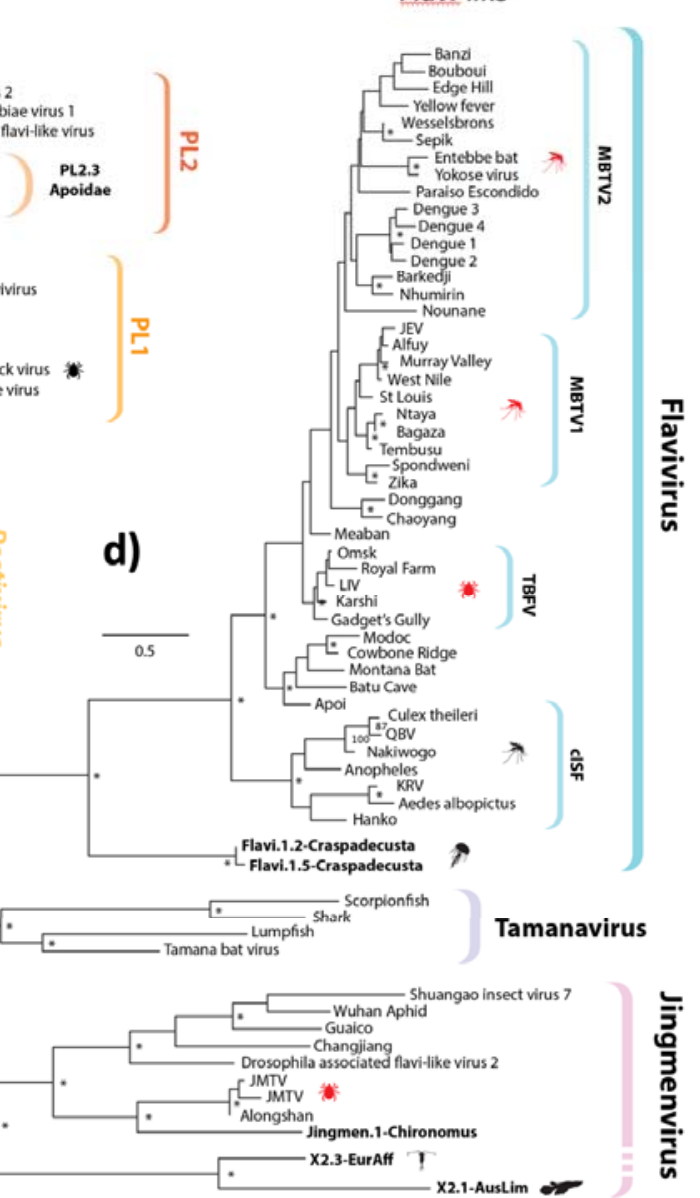

e)

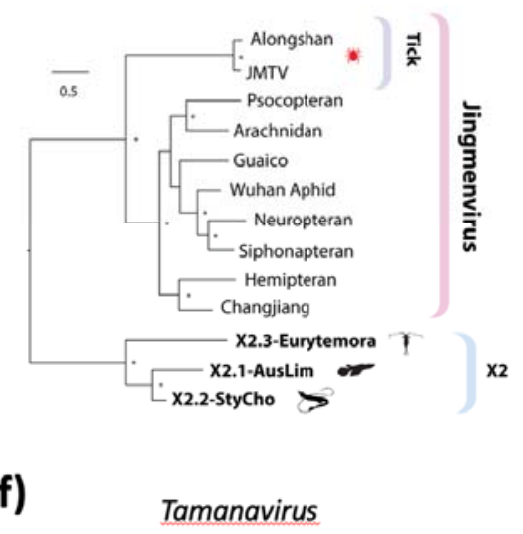

f)

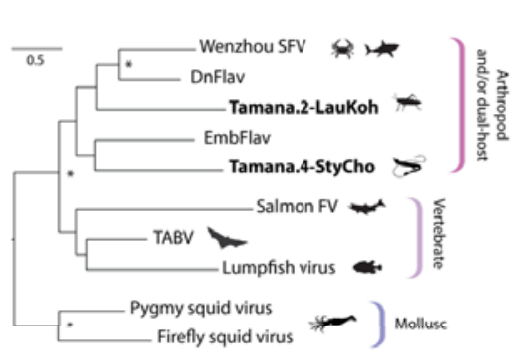


a)

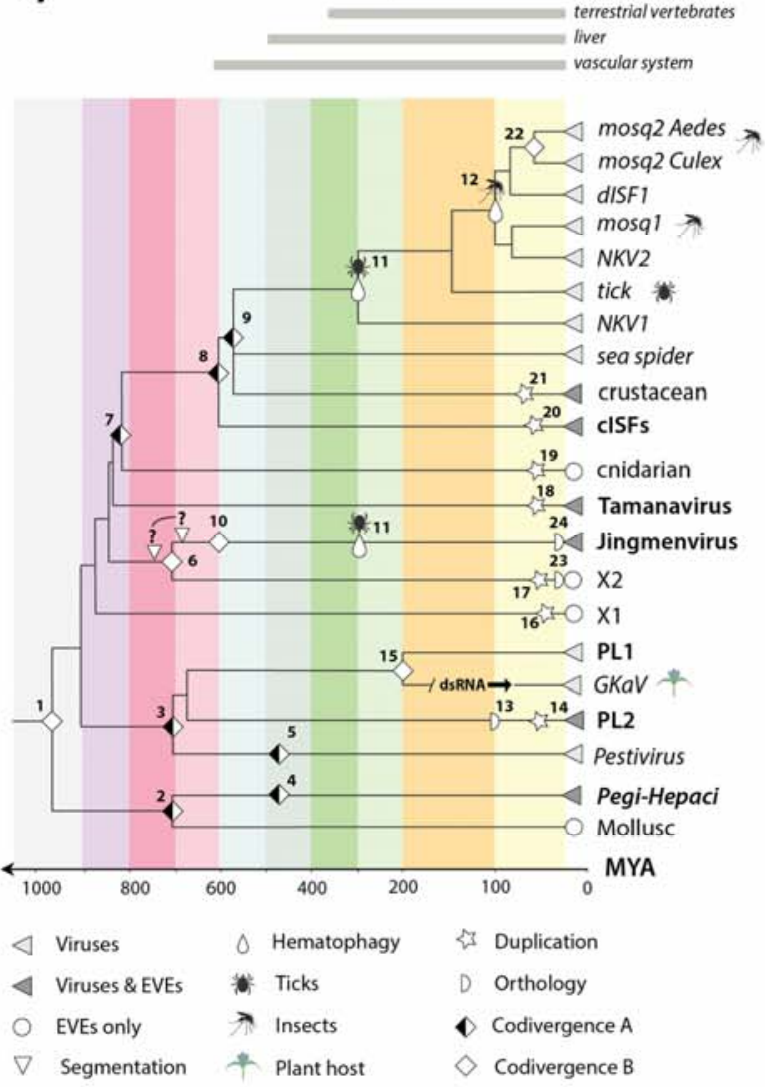

b)

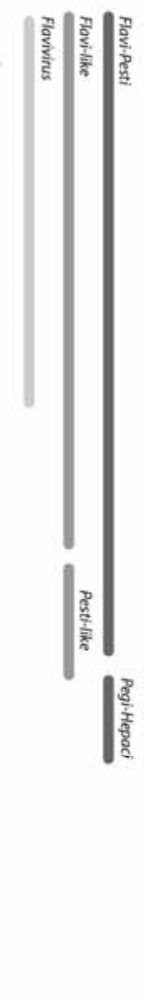

c)
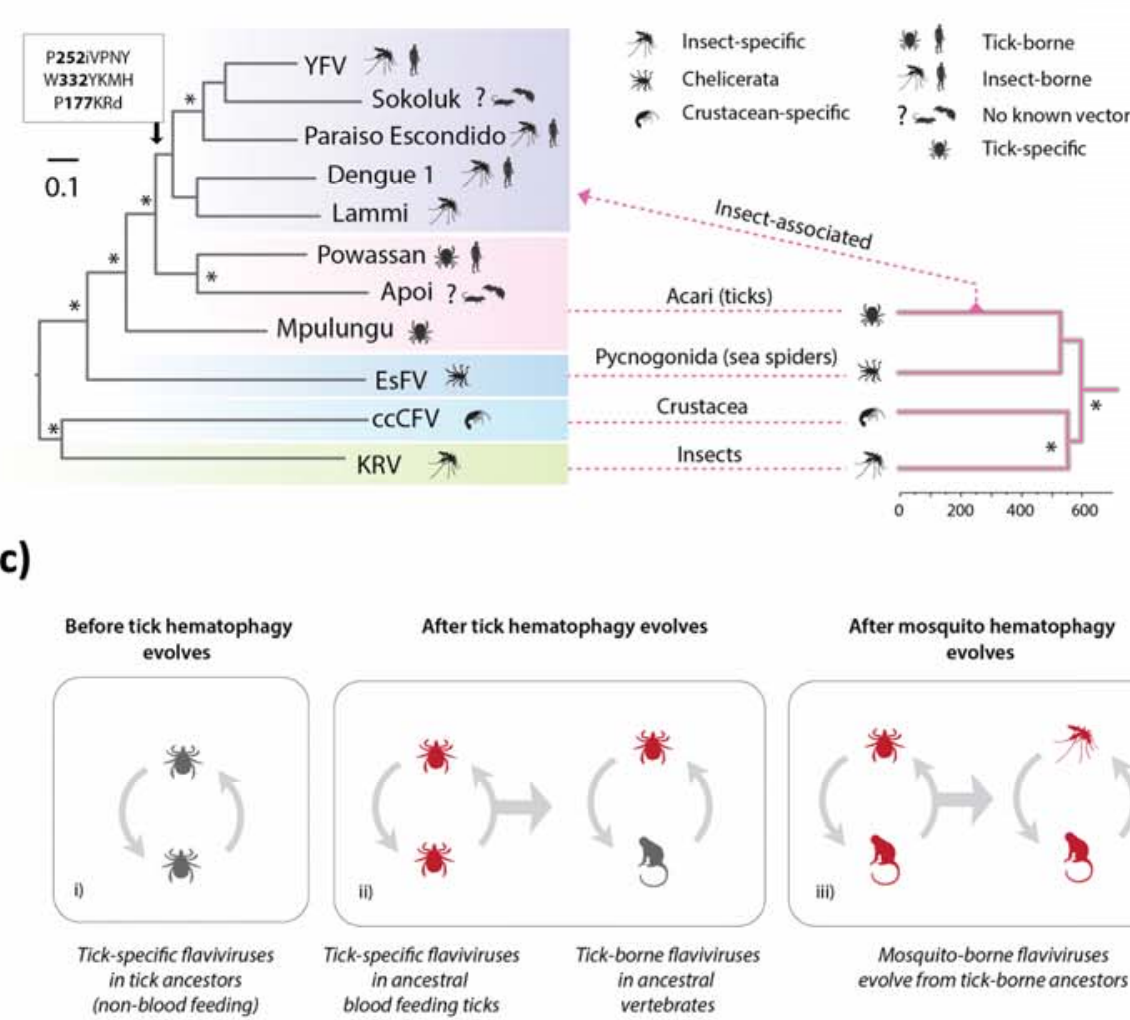

Before tick hematophagy evolves

After mosquito hematophagy evolves

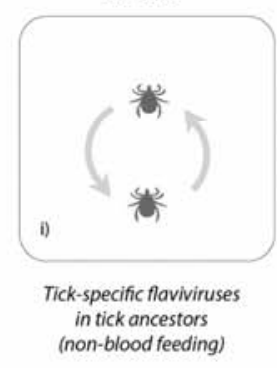

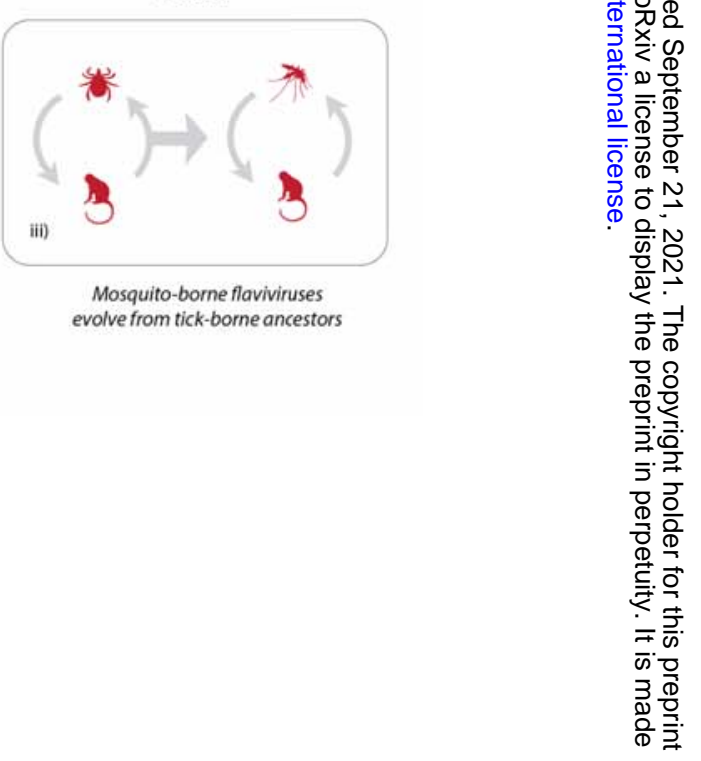

